\title{
A Semantic Information Retrieval Approach Based on Rough Ontology
}

\author{
Jun $\mathrm{Hu}^{1, *}$, Xinzhou $\mathrm{Lu}^{1}$ and Chun Guan ${ }^{2}$ \\ ${ }^{I}$ School of Software, Nanchang University, Nanchang, 330047, China; ${ }^{2}$ School Department of Computer Science, \\ Nanchang University, Nanchang, 330031, China
}

\begin{abstract}
With the emergence of the semantic web, ontology has attracted a great deal of attention in field of information retrieval. But the conceptual formalism supported by typical ontology is not sufficient for handling incomplete information that is confronted in the real world knowledge. To tackle this problem, a semantic information retrieval approach based on a rough ontology is proposed. Rough ontology in this paper is in the form of an ontology information system. Given a keyword based query, our approach infers the individuals and properties correlated to the query through a procedure of association searches in the rough ontology, and takes properties as equivalence relations to construct an approximation space of rough ontology. Afterward, an algorithm of computing similarity in rough ontology is presented, and approximation space is employed to compute similarity for ranking documents in semantic document indexing space. The proposed approach has been compared with two other information retrieval techniques, and the experiments conducted on CNKI collections, support the better efficacy which results from our approach.
\end{abstract}

Keywords: Association search, rough ontology, semantic information retrieval, similarity.

\section{INTRODUCTION}

As people realize the importance of information retrieval (IR) and as the web content is rapidly growing, more and more researchers have dedicated themselves to exploring more advanced retrieval approaches accordingly. In this paper a semantic information retrieval approach has been utilized which is based on the rough ontology; this is an exploration in the field of IR.

Currently, the traditional IR approaches are mostly based merely on keywords and are limited to string matching and link analysis. The existing information retrieval systems are mostly keyword-based and retrieve relevant documents or information by matching keywords. Keyword-based search, in spite of its merits of expedient query for information and ease-of-use, has failed to represent the complete semantics contained in the content and has let to the retrieval failure [1]. With the emergence of the semantic web, ontology has attracted a great deal of attention in the field of IR. Ontology is a formal explicit specification of a shared conceptualization, and it constitutes a backbone of the semantic web, facilitating a machine representation of information. It bridges an effective communication gap between the users and the machines. Therefore, ontology-based approaches have been put forward as one of the motivations for the semantic web in order to surmounting the limitations of keyword-based search. Unfortunately, the conceptual formalism supported by the ontology structure is not sufficient for handling imprecise, ill-structured or uncertain information confronted in real world knowledge. It is common knowledge now that

\footnotetext{
*Address correspondence to this author at Nanjing East Road, Nanchang, China. Postcard: 330047; Tel: 86-791-88305691;

E-mail: hujun@ncu.edu.cn
}

fuzzy set [2] and rough set [3] are the two major and widely applicable methods to process uncertain information. Thus, a positive use of fuzzy logic or rough set theory may be exploited in order to enhance the power of the semantic web. It has been shown that fuzzy logic allows bridging the gap between human-understandable soft logic and machinereadable hard logic. Indeed there has been a natural integration of fuzzy logic in ontology, in order to define a new theoretical paradigm called fuzzy ontology $[4,5]$. The fuzzy ontology is employed in order to improve the semantic IR by exploiting an additional knowledge hidden in entitiesdocuments relationships and also to enrich the semantics of the system after querying a database. However, these approaches still require the appropriate membership values to be assigned to evaluate the similarities between the concepts in a concept hierarchy. Meanwhile, the Rough set is also confronted with a new challenge, namely the vision of the semantic web. The Rough set theory incorporates with the existing ontology concepts and can provide possibilities to quantify a degree of accuracy of knowledge,. It can also a concept of rough ontology, which is an extended concept of the Rough set [6]. The unique advantage offered by the theory is that it enables us to use flexible information system in the form of rough ontology.

This paper proposes an approach to rough ontologybased on information retrieval which is meant for the exploitation of rough ontology, to support semantic search in document repositories. We propose an adaptation of the classical vector-space model to the index documents embedded with the individuals of ontology, which can represent the semantics of document more precisely. Furthermore, an algorithm for computing the similarities in the rough ontology has been presented. The degrees of the similarities are then used to re-rank the documents. 
The rest of this paper is organized as follows. An overview of the related work is given in Section 2. Section 3 presents the flow of our approach to rough ontology-based information retrieval. The experimental results are presented in Section 4. And the concluding remarks and future work are in the final section.

\section{RELATED WORK}

The related work to our approach comes from two main areas: ontology based IR and extensions of the ontology theory.

Knowledge Base (KB) approaches to ontology based IR use a reasoning mechanism and ontological query languages to retrieve instances. For example, OntoSCORM [7] is an ontology-based query expansion system for information retrieval, wherein the system employs description logic of ontology in order to infer relevant and similar concepts of queries. Onto Seek [8] is a system designed for content-based information retrieval. It combines an ontology-driven content-matching mechanism with moderately expressive representation formalism. Documents are treated either as instances or are annotates using the ontology instances [9]. Despite the fact that these works are focused on retrieving ontology instances, we are focused on retrieving the documents annotated with instances.

There are approaches combining both ontology based IR and vector space model. For instance, Rocha et al. [10] propose a search architecture that combines traditional search engine techniques with spread activation techniques that can be applied to a semantic model for a given domain. It can infer relations through a spread activation algorithm, making it possible to link concepts with semantic meaningful ontology instances in order to find related concepts in the ontology that are related to a given word, even if that word does not appear inside the concept. In our work, the query is also made through keywords that are mapped to existing concepts in the underlying ontology.

QuizRDF [11] is a system which combines traditional keyword searches with the possibility of querying and navigating through the RDF annotations of the resources when they exist. The RDF information is indexed together with the textual information of the resources. The resulting index makes it possible for the system to search for keywords inside both the textual information of the resource and its RDF annotations. The possibility of mixing traditional information retrieval with semantic information retrieval is a common aspect with our work.

Stojanovic et al. [12] propose an interesting approach for ranking query results using semantic information. It considers other important sources for determining the relevance of results to a query such as the structure of the underlying domains and the characteristics of the search process. The content of the information repository is used for searching in a similar way to the weight mapping techniques proposed in this paper. They calculate the relevance of a relation instance for the user's query using a measure that is similar to the specificity measure proposed in this paper. However, the measure proposed in their work is symmetric. The ranking proposed in their work also takes into consideration the path used by each inferred result in order to calculate its relevance to the user's query. In their work though, the query is expressed through instances of the ontology and not through keywords.

However, the conceptual formalism supported by the typical ontology may not be sufficient enough to represent the uncertainty of the information commonly found in many application domains due to the lack of clear-cut boundaries between concepts of the domains [13-18]. The issue of tackling this type of problems, has attracted world-wide attention for further research and development, resulting in various extensions to the original ontology theory and increasingly widening the fields of its applications.

One possible solution is to incorporate fuzzy logic into the ontology to handle the uncertainty data. Traditionally, fuzzy ontology, which is an extension of the domain ontology with crisp concepts, is generated and used for text retrieval and in search engines. Calegari et al. [19] proposed a fuzzy ontology based approach for improving the semantic documents' retrieval. It formally defines a fuzzy knowledge base and a special type of new non-taxonomic fuzzy relationship, called correlations that are assigned by experts, and then uses an information retrieval algorithm using an ObjectFuzzy Concept Network (O-FCN) which is involved in all steps of the algorithm which are word vector extensions, documents extractions and relevance calculations, in order to semantically enrich the results that are obtained. However, these approaches still require the appropriate membership values to be assigned to each occurrence of a term.

Another possible solution is to incorporate a rough set into the ontology. The Rough set theory works by exploring and exploiting the granularity structure of the data only. This is a major difference when compared with the fuzzy set theory which requires probability assignments and membership values respectively. Ishizu et al. [6] formulated a concept of rough ontology, which is an extended concept of the rough set, and define extended concepts of rough ontology. In this way, rough set theory using the concept of ontology enables us to use flexible information system in ontological description. Stuckenschim et al. [20] makes use of rough set-based ontology by putting restrictions on the ontological primitives in order to define its concepts.

Efforts demonstrate some of the potential of rough sets for information retrieval [21]. The model was applied by considering the domain's vocabulary (individual words and phrases) as the universe $U$ of objects. $R$ represented the equivalence relation defined by the term synonymy relationship and was used to create a partition of $U$ such that the terms within a class are synonyms with each other. The documents and queries, represented by vectors, were compared via their approximations in the approximation space. An interesting approach for computing rough similarity measures to compute document's overlaps is presented in [22]. Let S1 and S2 represent two subsets, which are the collections of weighted words. Let S1 represent the words in a retrieved document and S2 represent the words in a query. One would be interested to find the similarity between the query and the document in order to judge the relevance of the document with respect to the query. The approximations for a set $\mathrm{S}$ are computed as the union of the approximations 


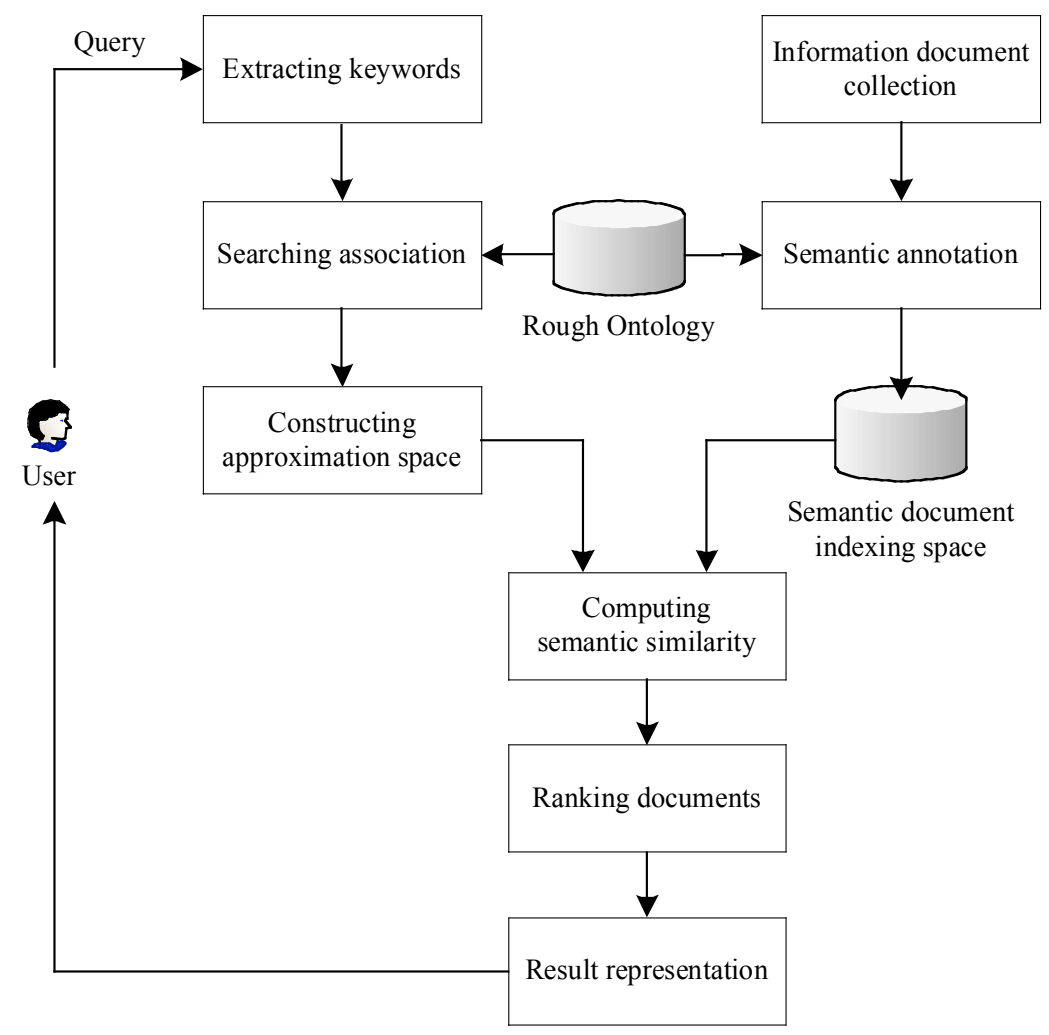

Fig. (1). The procedure of semantic information retrieval approach based on rough ontology.

of all the words occurring in it. These approximations are then used to find the similarities between two weighted set of words S1 and S2. They calculate the similarity between two subsets using a measure that is similar to the measure proposed in this paper. The similarity is calculated between individual sets and document sets are retrieved using a traditional keyword-based on retrieval method, as preformed in our work.

\section{A SEMANTIC INFORMATION RETRIEVAL APPROACH BASED ON ROUGH ONTOLOGY}

For retrieving information more efficiently in uncertain information spaces, a semantic information retrieval approach based on rough ontology has been presented to support the semantic search in document repositories. The steps of the proposed approach are shown as follows:

Step 1: A user inputs the initial information retrieval request by query.

Step 2: The system extracts keywords after receiving the initial information retrieval request.

Step 3: The system does an association search in rough ontology by keywords, and then returns individual sets and property sets correlated with the queries.

Step 4: The system uses property sets as an equivalent relation for the universal set $U$ to construct approximation space.

Step 5: The system utilizes the approximation space to search the semantic document space using keyword-based method, and then returns a list of documents, which has been annotated with semantic information of ontology.
Step 6: The system computes similarity of elements of the document set from Step5 and individual sets from Step4, and then returns a list of documents which is ranked by the degree of similarity.

The procedure for the semantic information retrieval approach based on rough ontology is highlighted in Fig. (1). When a user submits queries consisting of keywords to the system, the system can extract keywords. Furthermore, the system infers individual sets and property sets correlated to query through a procedure of association search mechanism in the rough ontology, and takes the property set as equivalence relation to construct an approximation space for the rough ontology. Afterward, the approximation space is employed to compute the similarities for the ranking documents in semantic document indexing space. The detailed algorithms are put forward in the following sections.

\subsection{Extracting Keywords}

When a user inputs the initial information retrieval request by query, the system extracts the most relevant words or phrases as keywords in the query.

\subsection{Searching Associations}

Association designates the relationship between one thing's object and another thing's object. The association among individuals may be direct or indirect. In individuals, the direct association means that two individuals are connected with a property directly. Otherwise, the indirect association is that two individuals are connected by a set of individuals and properties. The two kinds of associations play an important role in extracting information of interest, and are 
very helpful for the users for understanding the search results, especially on semantic web.

For example, the domain of a company contains the concept of "Manager", "Department" and "Staff". A search with two keywords may return the instance "Bill" which belongs to the concept "Manager" and the instance "Tom" which belongs to the concept "Staff". After these two instances are retrieved, users may want to know their associations. If fortunately, they have direct associations as the following triple: Bill has Staff Tom. So many existing semantic search systems can find the associations between them successfully. But the association is often indirect. Looking into the ontology, we may find that there is an indirect association: Bill works in "software development department", Tom work in "software development department". Jack and Jerry can be associated via "software development department".

This paper uses features of association relationship between individuals to infer keywords correlated with individuals and properties in the rough ontology. The procedure is shown as follows:

(1) Given a query, it is represented by a set of keyword $q$ $=\left\{k_{i}\right\}$. Search for individual is represented by $I_{q}$ and is related to $q$. Let $I_{k i}=\left\{i_{k i}\right\}$ denotes the set of individuals containing keyword $k_{i}$ as one of their sub strings. For that, it has the set of individual containing keyword q, $I_{q}=I_{k 1} \cup \ldots \cup I_{k i} \cup \ldots$.

(2) Infer the properties associated $i_{k i}$ and $i_{k j}$, where $i_{k i} \in I_{k i}, i_{k j} \in I_{k j}$. There are three different situations:

If individual $i$ contains keywords $k_{i}$ and $k_{j}$, then the association between $k_{i}$ and $k_{j}$ is viewed as Null Association.

If individuals' $i_{k i}$ and $i_{k j}$ are related by a property $p$, that is to say, the association between $i_{k i}$ and $i_{k j}$ is viewed as Direct Association; let the universal set of properties $p$ be viewed as $Q_{q}$.

If individuals $i_{k i}$ and $i_{k j}$ don't have any direct relationship, then our task is to find the indirect association between $i_{k i}$ and $i_{k j}$, in other words, there may exist a relationship path form $i_{k i}$ and $i_{k j}$. E.g., if $i_{k i}$ and $i_{i}$ are in direct association by the property $p_{a}$, which is represented as $\left(i_{k i}, p_{a}, i_{i}\right)$, similarly, if there is $\left(i_{i}, p_{m}, i_{j}\right)$ and $\left(i_{j}, p_{b}, i_{k j}\right)$, so, for the above three direct paths, an indirect relation path exists between $i_{k i}$ and $i_{k j}$, which is $p_{a}, i_{i}, p_{m}, i_{j}, i_{j}, p_{b}$. If individuals $i_{k i}$ and $i_{k j}$ are indirectly associated, then a set of individuals and a set of properties, which link $i_{k i}$ with $i_{k j}$, are represented as $I_{q^{\prime}}$ and $Q_{q^{\prime}}$ respectively.

(3) Obtain a set of individual $I_{0}=I_{q} \cup I_{q^{\prime}}$, and a set of property $Q_{0}=Q_{q} \cup Q_{q^{\prime}}$ related to queries.

\subsection{Constructing Approximation Space}

Constructing approximation space is an important aspect in applying the rough ontology to information retrieval. Rough set theory with existing ontology concepts to provide possibilities to quantify the degree of accuracy of knowledge. Rough set classifies objects using upper-approximation and lower-approximation defined on an indiscernibility relation which is a kind of equivalent relation. The lower ap- proximation is a description of the domain objects which are known with certainty to belong to the subset of interest, whereas the upper approximation is a description of the objects which possibly belong to the subset. Given equivalent relation between objects of individuals, result in indiscernibility relation of approximation space constructed by equivalent class. Each subset of universal set of rough ontology concept is classified by equivalent relation. Sometimes, for given a universal set $U$, some subsets may be completely classified, and others may be not. In the process of retrieval, the documents and queries can be represented as a subset corresponding the universal set, and classify them approximately and can compare them to each other for similarity using their approximations. Therefore, we can describe them more exactly.

\subsection{Indexing Semantic Documents}

After constructing of a rough ontology, the process of upgrading the actual documents in order to make them machine-understandable semantic documents according to the specified domain ontology is called the process of the semantic annotation. Semantic annotation solutions can be divided into manual, semi-automatic and automatic methods which endow classes and properties of ontology with instances. Finishing the work of semantic annotation and proper semantic document indexing space, can be constructed.

In this paper, on the premise of the fact that the documents have been annotated, we adapt the classical vectorspace model to index the documents. The given document $d_{j}$, is represented by a vector:

$\vec{d}_{j}=\left(w_{1, j}, w_{2, j}, \ldots, w_{n, j}, i_{1, j}, i_{2, j}, \ldots, i_{m, j}\right)$

$n$ denotes the total number of keywords in the document sets, $m$ denotes the total number of individuals, $w_{i, j}$ denotes the keyword $w_{i}$ 's weight in the document $d_{j}$ and $i_{k, j}$ denotes an individual $i_{k}$ 's weight in the document $d_{j}$.

For every keyword $w_{i, j}$, its weight could be calculated using $t f / i d f$ measure.

$w_{i, j}=$ freq $_{i, j} \times \log \frac{N}{n_{i}}$

where, freq $_{i, j}$ denotes $w_{i}$ 's frequency in the document $d_{j}, N$ is the total number of document set in $D, n_{i}$ denotes the number of documents where the keyword $w_{i}$ appears in document set $D$.

\subsection{Computing Similarity}

In this paper, we adopt the methods of approximation in order to represent the individual set $I_{0}$ and the document set $D_{q}$ related to the queries, where $D_{q}$ denotes a document set retrieved using a traditional keyword-based retrieval method in the semantic document indexing space, and then compute the similarities of $I_{0}$ and each element of $d_{j}$ in document set $D_{q}$.

The algorithm of computing similarity is presented as follows:

Step 1: Individual universal set in ROIS (Rough Ontology Information System) is partitioned into an equivalent 
class set, and the partitioning is done using the property set $Q$ as an equivalent relation which results in an approximation space $A S=\left(U, Q_{0}\right)$. If $i_{1}, i_{2} \in U$, where $i_{1}$ and $i_{2}$ are the same equivalent class sets, and $i_{1}$ and $i_{2}$ are indiscernible in an approximation space.

Step 2: Computing $Q_{0}$-upper approximation $Q_{0} I_{0}$ and $Q_{0}$-lower approximation $\underline{Q_{0} I_{0}}$.

Step 3: While (each element $d_{j}$ in document of set $D_{q}$ ) Computing $Q_{0}$-upper approximation $Q_{0} d_{j}$ and $Q_{0}$-lower approximation $\underline{Q_{0} d_{j}}$.

Step 4: While (each element $d_{j}$ in document set $D_{q}$ )

$$
\begin{aligned}
& \text { Computing } \overline{\operatorname{SIM}}\left(d_{j}, I_{0}\right)=\left|\frac{Q_{0} d_{j} \cap Q_{0} I_{0}}{Q_{0} d_{j} \cup Q_{0} I_{0}}\right|, \\
& \underline{\operatorname{SIM}}\left(d_{j}, I_{0}\right)=\mid \frac{\frac{Q_{0} d_{j}}{\overline{Q_{0} d_{j}} \cup \underline{Q_{0} I_{0}}}\left|\underline{\underline{Q_{0} I_{0}}}\right|,}{\operatorname{SIM}\left(d_{j}, I_{0}\right)=\overline{\operatorname{SIM}}\left(d_{j}, I_{0}\right)+\underline{\operatorname{SIM}}\left(d_{j}, I_{0}\right) .}
\end{aligned}
$$

Step 5: The document $D_{q}$ is ranked according to the level of similarity's degree in $\operatorname{SIM}\left(d_{j}, I_{0}\right)$.

\section{EXPERIMENTS}

The construction of the new ontology will encounter many difficulties. One reason is that, it is a huge and complicated work and it also needs the participation ofdomain experts. Another reason is that, there are many ontology tools, such as OntoEdit [23] and Protégé [24], which are available for aiding the construction of ontology, ontology construction still needs human effort. Consequently, as we are not aware of any publicly available document set pre-annotated with an ontology, we adopt the following steps to construct the data set and ontology which is particularly well adapted for the experiments.
First, it's free for us. 1,500 periodical metadata from the operating system category in the website CNKI can be used as the foundation for the experimental documents.

Secondly, we get an available ontology for the operation system represented in the OWL from SWOOGLE [25], which is an ontology search engine. However this ontology should be modified in order to fit the experiments, such as adding in the necessary classes and properties. Afterwards, we can use Protégé, which offer a facility of hierarchy viewing to support the users to build and edit the ontology in order to amend it. After the creation of the OWL ontology, documents are enriched with instances of classes. Eventually, this ontology contains 257 classes, 78 properties and 656 individuals.

Finally, we could index the documents and construct ontology information system.

Because of the limited scope of our data collection, we restrict the test queries to those which have at least 30 relevant documents in the data set. Based on a set of 10 test queries, we conducted a set of experiments to measure the performance of the three technologies: 1) rough ontology-based, 2) OntoSCORM, and 3) Lucene. Performance of these technologies is measured by F-measure and P@10 (Precision at top ten ranks). F-measure is the weighted harmonic mean of the precision and recall. It can be represented as following: Precision $=\frac{\mid\{\text { relevant documents }\} \cap\{\text { retrieved documents }\} \mid}{\mid\{\text { retrieved documents }\} \mid}$,
Recall $=\frac{\mid\{\text { relevant documents }\} \cap\{\text { retrieved documents }\} \mid}{\mid\{\text { relevant documents }\} \mid}$, F-measure $=2 \times($ Precision $\times$ Recall $) /($ Precision + Recall $)$.

Fig. (2). shows the results of the performance among these three technologies. The y-axis is an F-measure. As shown in Fig. (2), rough ontology-based technology outperforms the other two technologies in the F-measure, in all query sets from 1-10.

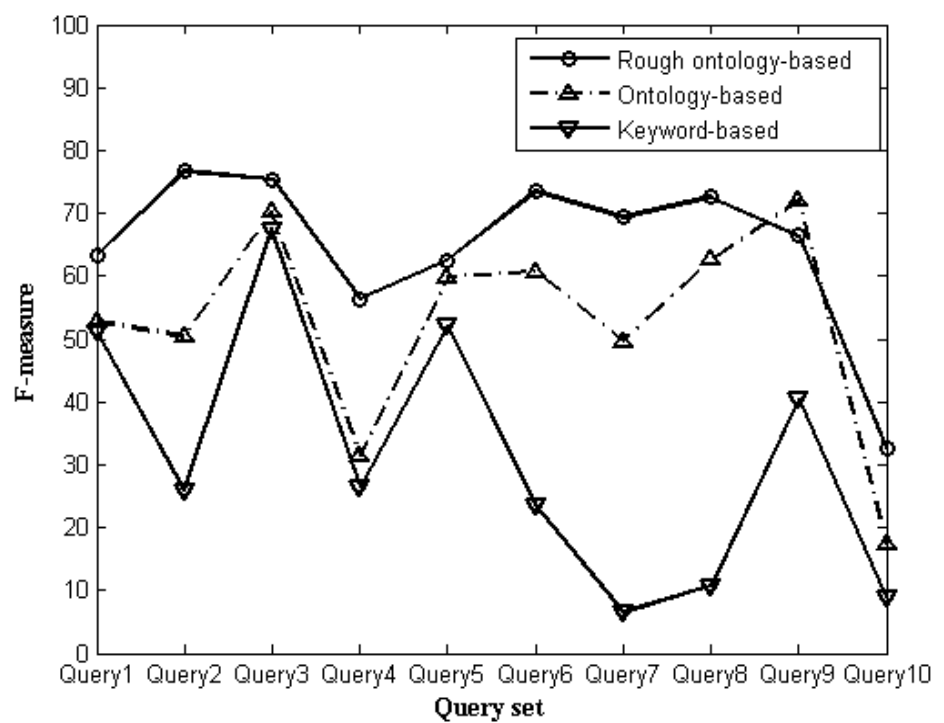

Fig. (2). An illustration of the F-measure among the three methods. 


\section{CONCLUSION}

In order to retrieve semantic documents more precisely, a rough ontology-based semantic information retrieval approach has been proposed in this paper. The approach is a hybrid information retrieval technology combining rough ontology with classical search technology and association search technology. Furthermore, a new algorithm for computing the semantic similarities in the rough ontology is presented. Taking keyword-based queries as input, the association search mechanism can infer individual sets and property sets correlated with the queries to from a rough ontology. Taking property sets as equivalence relation to construct an approximation space wherein a procedure of computing similarities between individual sets and document sets retrieved using a traditional keyword-based retrieval method is carried out. The degrees of the similarity are then used to rerank the documents. To examine whether our approach improves the retrieval performance or not, we compared our approach with the keyword-based method and the ontologybased query expansion method, and the initial results were good. In the future studies the issue of, making use of ontology inference mechanism and attribute reduction of rough ontology should be addressed. Our system has this issue that we are currently investigating in our ongoing work.

\section{CONFLICT OF INTEREST}

The authors confirm that this article content has no conflict of interest.

\section{ACKNOWLEDGEMENTS}

This work is supported by the National Natural Science Foundation of China (Grant No. 11226042) and the Science Research Foundation of Jiangxi Educational Committee (Grant No. GJJ12050).

\section{REFERENCES}

[1] M. Y. Chen, H. C. Chu, and Y. M. Chen, "Developing a semantic-enable information retrieval mechanism," Expert Syst. Appl., vol. 37, pp. 322-340, 2010.

[2] L. A. Zadeh, "Fuzzy sets," Inform. Control, vol. 8, pp. 338-353, 1965.

[3] Z. Pawlak, "Rough set," Int. J. Inf. Comp. Sci, vol. 1, pp. 341$3561,1982$.

[4] B. Fernando, and S. Umberto, "Aggregation operators for fuzzy ontologies," Appl. Soft Comput., vol. 13, pp. 3816-3830, 2013.

[5] Z. Fu, Z. M. Ma, and Y. Li, "Construction of fuzzy ontologies from fuzzy XML models," Knowl-Based Syst., vol. 42, pp. 20-39, 2013.
[6] S. Ishizu, A. Gehrmann, Y. Nagai, and Y. Inukai, "Rough ontology: Extension of ontologies by rough sets," Lecture Notes in Comput. Sci., vol. 4557, pp. 456-462, 2007.

[7] X. Hong, "Study on the Semantic of SCORM based on Knowledge Organization \& Retrieval," Beijing: Beijing University, 2006.

[8] N. Guarino, C. Masolo, and G. Vetere, "OntoSeek: content-based access to the web," IEEE Intell. Syst., vol. 14, pp. 70-80, 1999.

[9] A. Kiryakov, B. Popov, I. Terziev, D. Manov, and D. Ognyanoff, "Semantic annotation, indexing, and retrieval," J. Web Semantics, vol. 2 , pp. 45-79, 2005.

[10] C. Rocha, D. Schwabe, and M. P. Aragao, "A hybrid approach for searching in the semantic web," In: Proceeding of $W W W$ $A C M$, pp. 374-383, 2004.

[11] J. Davies, R. Weeks, and U. Krohn, "QuizRDF: Search Technology for the Semantic Web," Workshop on RDF \& Semantic Web Applications in Proc. WWW2002, Hawaii, USA, 2002.

[12] N. Stojanovic, R. Struder, and L. Stojanovic, "An Approach for the Ranking of Query Results in the Semantic Web," In: Proc. of $I S W C$, vol. 03 , pp. 500-516, 2003.

[13] S. Rossitza, T. Qiao, and S. Ivan, "Semantic-based information retrieval in support of concept design," Adv. Eng. Inform., vol. 25, pp. 131-146, 2011.

[14] M. Y. Pai, M. Y. Chen, H. C. Chu, and Y. M. Chen "Development of a semantic-based content mapping mechanism for information retrieval," Expert Syst. Appl., vol. 40, pp. 2447$2461,2013$.

[15] X. T. Zhang, X .Hou, X. F. Chen, and T. Zhuang, "Ontologybased semantic retrieval for engineering domain knowledge," Neurocomputation., vol. 116, pp. 382-391, 2013.

[16] T. Y. Tao, and M. Zhao, "An ontology-based information retrieval model for vegetables e-commerce," J. Integr. Agric., vol. 11 , pp. 800-807, 2012.

[17] S. Licia, and T. Roberto, "A novel semantic information retrieval system based on a three-level domain model," J. Syst. Softw, vol. 86, pp. 1426-1452, 2013.

[18] K. Efstratios, B. Christos, D. Theologos, and B. Nick, "Ontologybased sentiment analysis of twitter posts," Expert Syst. Appl., vol. 40, pp. 4065-4074, 2013.

[19] S. Calegari, E. Sanchez, "A Fuzzy Ontology-Approach to improve Semantic Information Retrieval," In: Proceedings of the Third ISWC Workshop on Uncertainty Reasoning for the Semantic Web, November 12, 2007.

[20] H. Stuckenschim, and U. Visser, "Semantic translation based on approximate reclassification," In: Proc. Workshop on Semantic Approximation, Granularity and Vagueness, Breckenridge, Colorado, USA, 2000.

[21] P. Srinivasan, "The importance of rough approximations for information retrieval," Int. J. Man-Machine Stud, vol. 34, pp. 657-671, 1991.

[22] P. Srinivasan, M. E. Ruiz, D. H. Kraft, J. Chen, and S. Kundu, "Vocabulary mining for information retrieval: Rough sets and fuzzy sets," Information Processing and Management, vol. 37, pp. 15-38, 2001.

[23] Y. Sure, S. Staab, M. Erdmann, J. Angele, R. Studer and D. Wenke, "OntoEdit: Collaborative ontology development for the semantic web,”, In: Proc. of ISWC2002, pp. 221-235, 2002.

[24] Protégé, http://protege.stanford.edu/

[25] Swoogle, http://swoogle.umbc.edu/

(C) Hu et al.; Licensee Bentham Open.

This is an open access article licensed under the terms of the Creative Commons Attribution Non-Commercial License (http://creativecommons.org/licenses/by-nc/3.0/) which permits unrestricted, non-commercial use, distribution and reproduction in any medium, provided the work is properly cited. 